\title{
A Resource-Constrained Approach to Implementing Analytics in an Institution of Higher Education: An Experience Report
}

\author{
John P. Buerck and Srikanth P. Mudigonda \\ Saint Louis University, USA \\ buerckjp@slu.edu
}

\begin{abstract}
Academic analytics and learning analytics have been increasingly adopted by academic institutions of higher learning for improving student performance and retention. While several studies have reported the implementation details and the successes of specific analytics initiatives, relatively fewer studies exist in literature that describe the possible constraints that can preclude an academic or learning analytics initiative from succeeding fully, meeting the criteria of success as defined by the stakeholders affected by such initiatives. Our aim in this article is to describe the constraints that precluded a successful completion of our analytics initiative and how we re-envisioned our approach and scope to achieve our primary goals while operating within the constraints and tools associated with our academic environment.
\end{abstract}

KEYWORDS: Learning analytics, academic analytics, learning management system, student retention

\section{INTRODUCTION}

The purpose of this article is to describe the our experience with our initiative in applying analytics to improve student performance and student retention, and situate this in the experiences and best practices reported in the literature pertaining to how academic and Learning Analytics initiatives have been undertaken by various academic institutions of higher learning.

Campbell, DeBlouis, and Oblinger explain that "Analytics marries large data sets, statistical techniques, and predictive modeling" and say that analytics "could be thought of as the practice of mining institutional data to produce 'actionable intelligence'" (2007, p. 42). When the context is an academic institution, and the goals are student success and retention, the application of analytics at an institutional level to achieve the above-stated goals is termed as "Academic Analytics" (AA) (Long \& Siemens, 2011). When the overarching goals of student success and retention are used as a focus within individual courses to improve student performance in individual courses, by measuring, collecting, analyzing, and reporting data related to student learning, participatory, and performance-related behaviours, then the particular analytics approach is termed "Learning Analytics" (LA) (Long \& Siemens, 2011). Thus, LA can be seen as an important component of an educational institution's AA initiative and that successful implementation of LA is crucial to the success of the institution's AA initiative. 
(2014). A Resource-Constrained Approach to Implementing Analytics in an Institution of Higher Education: An Experience Report. Journal of Learning Analytics, 1(1), 129-139.

According to Greller and Drachsler (2012), there are six dimensions related to an LA initiative that need to be addressed well in order for the LA initiative to be successful. They are: (1) stakeholders, consisting of various groups of individuals who will be affected by the LA initiative, such as students, instructors, administrators, staff and other personnel; (2) objectives, consisting of the specific stakeholders' goals that the LA initiative is intended to address; (3) data, derived from the actions and activities undertaken by students, instructors, administrators, and other personnel involved in the operations of the academic institution; (4) instruments, consisting of the theories related to the behaviours of various actors in an educational setting and how they affect the outcome(s) of interest, technologies consisting of hardware and software, including the analysis algorithms, reporting and visualization tools and formats, etc.; (5) external constraints, such as conventions, norms, and legal requirements pertaining to data privacy (e.g., the US Family Educational Rights and Privacy Act, FERPA), research ethics, time period for which the LA initiative needs to exist before its outputs can be seen as beneficial to the stakeholders; (6) internal limitations, such as the competencies of various stakeholders to participate effectively and take advantage of the tools and technologies made available to them through the LA initiative.

Reflecting on our LA-related efforts thus far, and viewing them through the perspective of the six dimensions provided by Greller and Drachsler (2012), we were able to understand the reasons why our analytics initiative, which we originally envisioned as an AA initiative, did not meet our expectations, and how our revised version of the LA has been able to achieve moderate levels of success. In the next section, we will use the perspective provided by Greller and Drachsler (2012) to provide two sets of analyses: (1) the shortcomings of our original AA initiative; (2) a revised version of the initiative, which can now be described as an LA initiative due to a revision of the goals and scope. The revised version has achieved moderate success.

\section{OUR ACADEMIC ANALYTICS AND LEARNING ANALYTICS INITIATIVES}

\subsection{Background}

A survey of extant literature related to academic and Learning Analytics revealed that academic institutions of higher learning have successfully deployed analytics at various levels in the institutional hierarchy successfully. For instance, the president of Arizona State University made analytics a central component in university-wide change in focus to improve student performance and retention, while also launching newer academic departments and programs and increasing the university's societal impact, successfully (Crow, 2012). Baylor University is cited as one of the pioneers in using analytics in support of student recruitment and admissions (Campbell et al., 2007). The University of Alabama, Sinclair Community College, and Northern Arizona University have used analytics in improving student retention by identifying under-performing students early and making necessary interventions to ensure that they improve their academic performance and graduate (Campbell et al., 2007). Another "large-scale success" of an Academic Analytics initiative is Purdue's Signals system, which is being used successfully across 
(2014). A Resource-Constrained Approach to Implementing Analytics in an Institution of Higher Education: An Experience Report. Journal of Learning Analytics, 1(1), 129-139.

several courses in various departments and academic programs, and has been converted successfully into a product that can be procured and implemented by other academic institutions as well (Arnold, 2010; Tally, 2010; Norris \& Baer, 2013).

Given the above-cited Academic Analytics initiatives that have been successful, our team decided to use a top-down approach for planning the various phases of the initiative and implementing them using an approach familiar to the initiative managers in the IT support services department. The impetus for our AA initiative came from higher-level administration officials in the IT support services and university administration departments, and two faculty members, who, in addition to teaching and researching, had responsibilities in department-level administration.

The higher-level and the department-level administrators realized that a cohesive, data-driven approach was needed to identifying students at risk of performing poorly and consequently either falling behind in their coursework or possibly leaving the university. By identifying such students and by providing them with necessary help and attention, it was expected that their academic performance and consequently their successful completion of their academic programs, could be achieved. From the IT support services side, the top-level officials realized that the large amounts of data being recorded in the learning management system (LMS), Blackboard, which the university uses, alongside the data related to student demographics and extra- and co-curricular activities, could be analyzed using tools related to Big Data and Analytics. Due to the convergence of these two sets of goals, an Academic Analytics initiative was sponsored by one high-ranking administrator and received support from high-ranking officials in IT support services. A team comprising IT personnel with expertise in data management, stewardship, administration of LMS and various student databases, an initiative manager, and two academic faculty members (the researchers/authors of this article) was formed.

According to the original initiative charter, a pilot version of the initiative would involve the collation of data of beginning-level students in the faculty members' department (computer information systems) drawn from their activities and performance recorded in the LMS, along with their demographic and extra-curricular activity data drawn from several disparate databases. These data would be cleaned, converted into a format amenable to data analysis after de-identification of individual student data (to ensure adherence to FERPA and other privacy-related requirements), and then models would be generated for creating profiles of students in an approach akin to that used in the Purdue Signals' initiative (Arnold \& Pistilli, 2012).

\section{ANALYSIS OF THE FIRST ATTEMPT USING THE GRELLER AND DRACHSLER PERSPECTIVE}

Our initial work in the implementation of Academic Analytics at our university did not meet our expectations for several reasons. The various initiative phases were either not initialized on time, or 
(2014). A Resource-Constrained Approach to Implementing Analytics in an Institution of Higher Education: An Experience Report. Journal of Learning Analytics, 1(1), 129-139.

those that were initialized experienced significant delays. We will now present an analysis for our approach by structuring it using the six-dimensional perspective provided by Greller and Drachsler (2012).

\subsection{Stakeholders}

Having a shared understanding of the initiative's goals and scope among various stakeholders affected by it, in particular among the initiative's sponsors and implementers, is a key determinant of an analytics initiative's success (Norris \& Baer, 2013; Crow, 2012). While we began with a common understanding of the scope of the initiative and its expected schedule, over the course of the pilot phase, deviations from the expected schedule led to a reallocation of priorities by various stakeholders, due to the constraints under which they were operating. This modification resulted in further deviations from the expected schedule, culminating in a disbanding of the initiative team and termination of the initiative in its original form.

\subsection{Objectives}

The primary objective of the pilot phase of the initiative was to develop a comprehensive infrastructure that included automated procedures for obtaining data from multiple data sources (student demographics and extra-curricular activities databases, LMS databases), removing personally identifiable data, and converting the data into a format amenable to analysis. The next step was to create statistical models able to profile students based on their background data, their curricular data (behavioural and performance data), and their extra-curricular data, e.g., their participation in various campus activities, their residency, and so on using an approach similar to that used in the Purdue Signals initiative (Arnold \& Pistilli, 2012). The generation of actionable information presented in a form easily understood by students, their advisors, the faculty, and high-level administrators was the goal. In hindsight, and in comparison to the initiative schedules reported from Academic Analytics initiatives of other institutions, we realized that our schedule did not account for the amount of work required for completing all the tasks while requiring access to the personnel with the necessary skills and expertise needed for completing all the tasks.

\subsection{Data}

The data in individual databases was stored in tables whose naming conventions were unique to each vendor. Making sense of the metadata and determining which tables in which databases contained potentially meaningful and useful data was a stupendous task requiring a significant amount of time and collaboration among various departments including IT support services and the office of student records. Additionally, ensuring compliance to various privacy-related guidelines and laws was also important. The significance of these factors, and the amount of time, effort, and human expertise required to address the data-related issues cannot be understated. However, for a team undertaking an Academic Analytics 
(2014). A Resource-Constrained Approach to Implementing Analytics in an Institution of Higher Education: An Experience Report. Journal of Learning Analytics, 1(1), 129-139.

initiative for the first time, the magnitude of the resources required were not readily apparent at the start of the initiative.

\subsection{Instruments}

Personnel who have hands-on experience with multiple vendors' database conventions, in addition to the specific naming and design conventions followed by the departments in charge of data and information technologies at an academic institution will play a crucial role in determining optimal approaches for preparing datasets ready for analyses. While the researchers were well conversant in using statistical methods for analyzing data and producing profiles of students, the initiative was hamstrung by a lack of availability of data management experts who could devote the amount of time necessary to produce the datasets in a form that the researchers could use on an ongoing basis. The researchers' attempts to make sense of the metadata combined with obtaining the necessary clearances to access the necessary data were also constrained due to their teaching and departmental administration tasks. An additional hurdle was the difficulty associated with the inter-weaving of data from multiple sources to use in the data analyses. Thus, the few analyses that were run produced results that were not surprising. For instance, in analyzing student data from a course in Computer Ethics, we found that students' access of various learning materials and of the grade book were positively associated with their final score. While this finding is consistent with the expectation that students who consistently obtain the learning materials and look at their performance and feedback are more studious and consequently more likely to perform well, it does not provide any insight into the thought patterns of those who are not performing well. Or as Strader and Thille put it, "students' knowledge state is a blackbox to the instructor" (2012, p. 205). As such, the model that resulted from analyzing the small sample dataset did not produce any actionable information.

\subsection{External Constraints}

There were relatively few external obstacles faced by our team since our existing university policies ensured compliance with all regulatory requirements related to student privacy. Additionally, by obtaining approval for an Institutional Review Board, we ensured that enough safeguards were in place that current students would not be adversely affected by our research outcomes and that the uncertainty associated with the "pay-off" of the initiative for current students in the future would be acceptable.

\subsection{Internal Constraints}

The biggest constraints encountered have been internal. We lacked enough personnel with the necessary expertise to produce the needed datasets in a timely manner. Additionally, several of the existing experts did not have the cross-domain expertise needed to create an automated process that produced the necessary datasets to be analyzed. Furthermore, the specific technology-related and 
(2014). A Resource-Constrained Approach to Implementing Analytics in an Institution of Higher Education: An Experience Report. Journal of Learning Analytics, 1(1), 129-139.

policy-related mechanisms for producing meaningful reports that can be used to take the actions necessary for improving student participation, performance, and thereby retention, required analysis and development of tools and policies. These, too, required time and human expertise that were in short supply. Thus, the original schedule for implementation of all aspects of the pilot project could not be met.

Subsequent to the termination of the initiative in its original form, the researchers redefined the scope of the initiative, with a goal of achieving a few noticeable results at the departmental level in the form of improved student retention and performance. In the next section, the new version of the initiative will be analyzed using, once again, the six dimensional perspective provided by Greller and Drachsler (2012).

\section{PROJECT REDUX: A REVISION OF SCOPE AND APPROACH}

Having analyzed our first attempt at incorporating analytics at an institutional level, which can best be described as an Academic Analytics initiative (Long \& Siemens, 2011), we determined that the chances of using analytics successfully within our department is possible if we could define the scope of the initiative by explicitly taking into account the various constraints associated with time, expertise, availability of technologies, and data that constitute the primary components of an analytics initiative. Fortuitously, during the time when we were planning the next attempt at initiating an analytics initiative, the LMS underwent a version upgrade. In the new version of Blackboard (version 9.1, Service Pack 11), a "Retention Center" functionality ${ }^{1}$ is available. The functionality provided by the Retention Center allows an instructor or a course-builder to set alerts that are triggered when a student's performance level, activity level (accessing the course), or involvement level (participation in various discussion fora, completion of various evaluative components) fall below certain preset threshold values. This functionality can be used by an instructor, a course-builder, or a teaching assistant to view, via a dashboard, the overall participation and performance of all the students in a course and take the necessary intervention steps. Details of how we are leveraging this functionality, and using it in conjunction with a few other changes to our LMS-based course websites and departmental policies will be explained using the six dimensions.

\subsection{Stakeholders}

The stakeholders now are the students, the department chair, the assistant chair, the advising staff, the instructors, and the dean. Prior to the upgrade to the new version of the LMS, instructors had to monitor each aspect of student activity and performance levels manually and send an early warning to their academic advisors who would then work with the student, in conjunction with the instructor and the department chair, if necessary, to help the student improve his or her performance in the course. The old version of the early warning system was seen as tedious; because of this perception, not all faculty

1 http://goo.gl/nopcTe 
(2014). A Resource-Constrained Approach to Implementing Analytics in an Institution of Higher Education: An Experience Report. Journal of Learning Analytics, 1(1), 129-139.

members used it often. The new version of the early warning system, which is integrated into the LMS via the Retention Center dashboard (a) reduces the number of steps that an instructor has to take to identify students who have fallen below the set thresholds for involvement and performance in the course, and (b) simplifies the process of sending an early warning. In their report on the determinants of success of the Purdue Signals system, Tanes, Arnold, King, and Remnet (2011) remark that reluctance on the part of instructors using the system stemmed from a lack of understanding of how the system could benefit them and not knowing the best practices related to its use. Based on this finding, and on feedback from faculty members not using the older early warning process optimally, we decided to implement the Retention Center-based early warning system in a select few courses, with the aim of determining which approaches seem to work best. The expected consequences would be a reduction in the amount of overhead associated with identifying students who need intervention and the provision of necessary information and feedback in a timely manner. Simultaneously, we have informed other faculty members about the availability of this system and our pilot initiative, and are conferring with them to obtain their input, and thus their buy-in, on how best to use the system to benefit our students, while being cognizant of any additional workload this might create.

\subsection{Objectives}

Our first objective was to identify the procedures that an instructor, the departmental administrators (chair and assistant chair), and the advising staff have to follow to ensure that intervention activities that encourage help-seeking and involvement on the part of students are optimized. This objective is consistent with the determination of similar procedures in the Purdue Signals system (Tanes et al., 2011). Our second objective was to improve communication with students about their participation and performance in the course and guide them to helpful resources (e.g., online and in-person tutoring and writing services).

Quicker and more informative communication with students is achieved by periodic monitoring by the instructor of the Retention Center dashboard to learn about student performance and then contacting students, via the Retention Center, regarding specific areas where they are lagging. Where needed, instructors get in touch with advisors to determine if an intervention requires a more personal approach (e.g., help with issues related to financial aid, overall course load, and so on) is needed. In our university, this is a continuation of existing departmental procedures. The provision of detailed feedback on each evaluative component has been implemented via rubrics that include detailed descriptions of criteria used in the evaluation of each component and comments on the extent to which a student's work addresses each criterion. Based on the anecdotal evidence, in the form of verbal feedback from the students, it appears that using component-specific rubrics and the Retention Center are meeting the intended objectives. More specifically, quantitative data will be obtained via end-of-term evaluations by students of various aspects of the courses. 
(2014). A Resource-Constrained Approach to Implementing Analytics in an Institution of Higher Education: An Experience Report. Journal of Learning Analytics, 1(1), 129-139.

Complementary to the above-described objectives, we are redesigning the navigational and organizational scheme of our course websites on the LMS by following the guidelines specified by Quality Matters. ${ }^{2}$ Based on the feedback we received from students during previous academic terms, we believe that by using a standard design template across all courses on the LMS, the cognitive burden experienced by students while navigating a course website is minimized when all the courses have the same navigational scheme and organization of course materials into various sections and folders. The changes to website organization will be implemented in the next academic term.

By grounding our LA initiative in best practices reported in the literature, using well-designed, consistent online course websites, in conjunction with timely intervention actions that encourage help-seeking and involvement by our students, we expect to see improved student performance and retention. The "success" of our initiative will be determined by: (a) our mastery of the functionality and associated options provided by the Retention Center; (b) the development of a set of operational procedures, based on our observations in using the Retention Center, in determining the optimal actions that will lead to an increase in student success and retention; (c) an actual increase in student success and retention, determined by comparing the performance and number of drops $^{3}$ in courses where the Retention Center is currently used, with the performance and number of drops in the same courses offered during the same time period in previous academic years; (d) a survey of students who have interacted with course websites built using the new set of guidelines to determine their perceptions of ease of use. When the approach from this pilot, defined to span the current academic year, is applied to other courses offered through our department, we intend to collect more data to determine student perceptions on using multiple course websites that have the same "look and feel" in terms of navigational elements and organization of course materials into different folders and sections.

\subsection{Data}

As stated previously, the lack of actionable information, derived from data available in a form amenable to analysis, was one of the primary factors affecting the success of our previous Academic Analytics initiative. In the new iteration of our initiative, the information needed to take the necessary interventions is processed and made available in an intuitive format by the LMS itself with no additional work, other than accessing the Retention Center, by those who need the information. Thus, in the current form of our initiative, all the data issues that constrained us previously have been obviated. At the end of the current academic term, we will analyze the feedback provided by students in the two introductory courses in computer information systems that are implementing the LA activities. These data will be used in conjunction with the notes we are keeping on the various actions taken by the instructors in the courses, along with the feedback provided by the instructors on their use of the Retention Center and the rubrics for communicating with the students regarding their participatory

\footnotetext{
2 https://www.qualitymatters.org/rubric

A drop is said to occur in a course when a student unregisters from the course.
} 
(2014). A Resource-Constrained Approach to Implementing Analytics in an Institution of Higher Education: An Experience Report. Journal of Learning Analytics, 1(1), 129-139.

behaviours and performance in the courses. Additionally, we will also compare the data from the current term related to student retention and performance in these courses, and compare it with data from the same courses in previous academic terms to determine whether there have been any changes in the retention and performance of students in these courses. Based on our analysis, we will determine the changes, if any, needed to our current operational procedures and expand the LA activities to a selected set of additional courses.

\subsection{Instruments}

In our new LA initiative, the instruments necessary for processing the data and viewing the information in a useful form are embedded within the LMS. Additional tools that aid in communication and providing feedback — for example email and video/telephone conferencing — have been in use for a long time, so no additional cognitive or time-related constraints are present.

\subsection{External Constraints}

As in the previous version of our analytics initiative, currently no external constraints affect our initiativerelated activities.

\subsection{Internal Constraints}

In the current version of our analytics initiative, constraints on technical expertise, which affected our previous pilot project, are absent. While learning to use the Retention Center functionality in an effective manner requires some additional time and effort, it is not imposing too big a burden, so we expect to continue with the initiative.

\section{CONCLUSIONS AND FUTURE DIRECTIONS}

As with any new project in an educational enterprise, this project encountered a few challenges. These challenges were, however, easy to overcome, as the goal of the project is to develop processes that help students overall to graduate successfully. In this section, we highlight the key challenges that were encountered and are being addressed by the team.

We learned from our two attempts at using analytics in an academic setting for improving student performance and retention that a top-down approach to Academic Analytics may not always work. A bottom-up approach, based on careful consideration of the functionality available in existing tools, can work when it is augmented by complementary communication and support procedures.

Our first attempt at Academic Analytics was guided by a schedule that proved untenable because we had not fully taken into account the diversity and complexity of activities to be completed, and the skills and 
(2014). A Resource-Constrained Approach to Implementing Analytics in an Institution of Higher Education: An Experience Report. Journal of Learning Analytics, 1(1), 129-139.

knowledge required of the team assigned to complete them. In our second attempt, our focus has been on student participation and performance in individual courses, by relying on the "Retention Center" functionality available in the LMS used in conjunction with communication and operational procedures. Consistent with findings reported in the literature (e.g., Tanes et al., 2011), these procedures are directed towards increasing students' help-seeking and participatory behaviours, with an expected improvement in their performance in coursework and consequently in the successful completion of their academic programs of study.

Based on our experiences, we believe that academic institutions intending to undertake AA and LA initiatives need not start with an all-out approach requiring the deployment of tools and analytical procedures subsumed under the Big Data paradigm (see e.g., Barton \& Court, 2012). Rather, they can use a multi-phased approach where they undertake small LA initiatives centred around one or a few courses, utilize existing LMS-based tools to determine, given the current constraints and technologybased affordances, the optimal set of communication, intervention, and help-providing procedures so that student performance and retention are maximized. Then, based on what they have learned through this experience, they can determine the next steps needed for scaling their initiative to encompass more courses, and eventually determine a path for a more complete implementation of LA, and eventually AA across their academic institution.

As for our own LA initiative, in the immediate future, we expect to expand the LA activities to a few other courses in the computer information systems department after making any changes deemed necessary based on an evaluation of the data ${ }^{4}$ collected from the courses where the LA activities have been introduced during the current term.

\section{REFERENCES}

Arnold, K.E. (2010). Signals: Applying academic analytics. Educause Review Online. Retrieved from http://www.educause.edu/ero/article/signals-applying-academic-analytics

Arnold, K.E., \& Pistilli, M.D. (2012) Course signals at Purdue: Using learning analytics to increase student success, Proceedings of the 2nd International Conference on Learning Analytics, 29 April-2 May 2012, Vancouver, British Columbia, Canada.

Barton, D., \& Court, D. (2012). Making advanced analytics work for you. Harvard Business Review, 90(10), 78-83.

Campbell, J.P., DeBlouis, P.B., \& Oblinger, D.G. (2007). Academic analytics: A new tool for a new era. Educause Review Online, 42(4), 40-57.

Crow, M.M. (2012). "No more excuses": Michael M. Crow on analytics. Retrieved from http://www.educause.edu/ero/article/no-more-excuses-michael-m-crow-analytics

\footnotetext{
${ }^{4}$ See description in the Data subsection that precedes this section. 
(2014). A Resource-Constrained Approach to Implementing Analytics in an Institution of Higher Education: An Experience Report. Journal of Learning Analytics, 1(1), 129-139.

Greller, W., \& Drachsler, H. (2012). Translating learning into numbers: A generic framework for learning analytics. Educational Technology \& Society, 15(3), 42-57.

Long, P.D., \& Siemens, G. (2011). Penetrating the fog: Analytics in learning and education. Educause Review Online. Retrieved from http://www.educause.edu/ero/article/penetrating-fog-analyticslearning-and-education

Norris, D.M., \& Baer, L.L. (2013). Building organizational capacity for analytics. Educause Report. Retrieved from http://www.educause.edu/library/resources/building-organizational-capacityanalytics

Tally, S. (2010). Purdue-developed student intervention tech available nationally. Purdue University News Service. Retrieved from http://www.purdue.edu/newsroom/general/2010/101011CampbellCourseSigna.html

Strader, R., \& Thille, C. (2012). The open learning initiative: Enacting instruction online. In D.G. Oblinger (Ed.), Game Changers: Education and Information Technologies (pp. 201-213). Educause.

Tanes, Z., Arnold, K.E., King, A.S., \& Remnet, M.A. (2011). Using signals for appropriate feedback: Perceptions and practices. Computers and Education, 57(4), 2414-2422. 